\title{
Validation of suitable internal control genes for expression studies in aging
}

\author{
M. Zampieri ${ }^{\text {a }}$, F. Ciccarone ${ }^{\text {a }}$, T. Guastafierro ${ }^{\text {a }}$, M.G. Bacalini ${ }^{\text {a }}$, R. Calabrese ${ }^{\text {a }}$, M. Moreno-Villanueva ${ }^{\text {b }}$, \\ A. Reale ${ }^{a}$, M. Chevanne ${ }^{c}$, A. Bürkle ${ }^{b}$, P. Caiafa ${ }^{a, *}$ \\ a Department of Cellular Biotechnologies and Hematology, II Faculty of Medicine and Surgery, "Sapienza" University of Rome, Viale Regina Elena 324,00161 Rome, Italy \\ ${ }^{\mathrm{b}}$ Molecular Toxicology Group, Department of Biology, University of Konstanz, Konstanz, Germany \\ ${ }^{\mathrm{c}}$ Department of Experimental Pathology and Oncology, University of Florence, Florence, Italy
}

\section{A R T I C L E I N F O}

\section{Article history:}

Received 27 August 2009

Received in revised form 14 December 2009

Accepted 20 December 2009

Available online 28 December 2009

\section{Keywords:}

Aging

Housekeeping genes

GUSB

Real-time PCR normalization

PBMC

\begin{abstract}
A B S T R A C T
Quantitative data from experiments of gene expression are often normalized through levels of housekeeping genes transcription by assuming that expression of these genes is highly uniform. This practice is being questioned as it becomes increasingly clear that the level of housekeeping genes expression may vary considerably in certain biological samples. To date, the validation of reference genes in aging has received little attention and suitable reference genes have not yet been defined. Our aim was to evaluate the expression stability of frequently used reference genes in human peripheral blood mononuclear cells with respect to aging. Using quantitative RT-PCR, we carried out an extensive evaluation of five housekeeping genes, i.e. $18 \mathrm{~s}$ rRNA, ACTB, GAPDH, HPRT1 and GUSB, for stability of expression in samples from donors in the age range 35-74 years.

The consistency in the expression stability was quantified on the basis of the coefficient of variation and two algorithms termed geNorm and NormFinder. Our results indicated GUSB be the most suitable transcript and $18 \mathrm{~s}$ the least for accurate normalization in PBMCs. We also demonstrated that aging is a confounding factor with respect to stability of $18 \mathrm{~s}$, HPRT1 and ACTB expression, which were particularly prone to variability in aged donors.
\end{abstract}

(c) 2009 Elsevier Ireland Ltd. All rights reserved.

\section{Introduction}

Quantitative PCR is the most powerful tool used to monitor changes in gene expression which, although sometimes very small, have relevance for the understanding of biological and molecular mechanisms. However the identification of reference genes, whose level of expression should not vary during cell growth, differentiation and transformation or in response to experimental treatment is becoming increasingly important. In recent years a number of studies have been focused on this problem (de Jonge et al., 2007; Dheda et al., 2004; Goidin et al., 2001; Huggett et al., 2005; Pfaffl and Hageleit, 2001; Ross et al., 2000; Vandesompele et al., 2002).

Aging is a physiological process associated with transcriptional deregulation of some genes and this is often correlated to changes of the DNA methylation pattern. DNA methylation has to be considered among the various factors that contribute to aging (Fraga, 2009; Fraga et al., 2007; Gronbaek et al., 2007). Studies carried out in homozygotic twins (HZ) showed that their genomes are epigenetically different (Fraga et al., 2005). In particular, while

\footnotetext{
* Corresponding author. Tel.: +39 0 649976530; fax: +39 0644231961.

E-mail address: Caiafa@bce.uniroma1.it (P. Caiafa).
}

young pairs were epigenetically similar, the old ones were clearly dissimilar. In addition $\mathrm{HZ}$ twins, who had spent a long period of their lives apart, showed differences in phenotype that were often associated with differences in their medical histories (Calvanese et al., 2009; Feinberg, 2007; Fraga et al., 2007). All this demonstrates that gene expression is age-dependent, and, as concerns the expression of housekeeping genes, aging is comparable to a premalignant situation in which methylation spreads towards the promoter (Gronbaek et al., 2007). When the cytosines located within the promoters become methylated, transcription is blocked, i.e. the genes affected are down regulated. In addition, diet and/or lifestyle can affect the maintenance of methylation pattern in aging (Burdge et al., 2007; Campisi et al., 2001; Delage and Dashwood, 2008; Feil, 2006; Finkel and Holbrook, 2000; Ghoshal et al., 2006; Grube and Burkle, 1992; Grummt and Ladurner, 2008; Herceg, 2007; Jaenisch and Bird, 2003; Liu et al., 2007; Migliore and Coppede, 2008; Petronis et al., 2003; Sutherland and Costa, 2003; Waterland, 2009).

Thus, to perform research on the aging process, the identification of control genes in humans is necessary as data available concern the mouse only (Bahar et al., 2006; Boda et al., 2009; Sieber et al., 2008; Somel et al., 2006; Tanic et al., 2007; Touchberry et al., 2006). 
In this study we evaluated the commonly used housekeeping genes 18s rRNA, glyceraldehyde 3-phosphate dehydrogenase $(G A P D H)$, and beta-actin as well as hypoxanthine guanine phosphoribosyl transferase 1 (HPRT1) and beta-glucuronidase (GUSB), as potential control genes for the influence of aging in peripheral blood mononuclear cells (PBMCs) obtained from human individuals from 35 to 75 years of age. Our aim was to select, among the above transcripts, mRNAs suitable for normalization of quantitative RTPCR data. Expression changes within the samples and between differentially aged groups of the samples were investigated to select the most stable reference gene showing the least variation of expression.

The five housekeeping genes transcripts chosen were selected as they have different physiological roles - ribosome component (18s rRNA), carbohydrates metabolism (GAPDH), cytoskeleton $(A C T B)$, metabolism of nucleotides (HPRT1) and catabolism of complex carbohydrates (GUSB) - thus the risk of the aging process affecting all of the genes tested is minimized. In addition each gene evaluated in our study has been proposed as a suitable control gene in at least one biological condition (Bas et al., 2004; Silver et al., 2008; Valente et al., 2009). In particular the suitability of GAPDH, $A C T B$ and GUSB for accurate normalization of gene expression with respect to aging has been demonstrated in the mouse white blood cells (Sieber et al., 2008).

\section{Materials and methods}

\subsection{Blood donors}

We analyzed venous blood samples obtained from apparently healthy volunteers representing 8 age groups (35-39, 40-44, 45-49, 50-54, 55-59, 60-64, 65-69, 70-74 years), with each group composed of five individuals (40-60\% males). Ethical clearance had been obtained by the University of Konstanz Ethics Committee.

\subsection{Samples collection}

The blood samples from participants were drawn into a plastic syringe containing sodium citrate. Peripheral blood mononuclear cells (PBMCs) were obtained by gradient centrifugation through "Lymphoprep" separating solution (Axis-Shield). Aliquots of cells (about $3 \times 10^{6}$ cells $/ \mathrm{mL}$ ) were frozen in RPMI medium containing $40 \%$ FCS, 20\% dimethyl sulfoxide and stored in liquid nitrogen until required. Before use, cells were gradually thawed by incubation at $37{ }^{\circ} \mathrm{C}$ followed by dropwise addition of RPMI containing 10\% FCS to a final dilution of $1: 20$.

\subsection{RNA extraction and cDNA synthesis}

Isolation of total RNA (from $3 \times 10^{6}$ cells) was performed using RNeasy Mini Kit (Qiagen) according to the manufacturer's instructions and subjected to DNase I digestion using RNase-free DNase (Qiagen). RNA concentration and purity were evaluated by measuring absorbance at 230, 260 and $280 \mathrm{~nm}$, respectively. Quantifications were repeated three times for each sample. Integrity of the RNA was verified by gel electrophoresis of $\sim 1 \mu \mathrm{g}$ RNA on a $1 \%$ agarose-TAE gel containing ethidium bromide. In intact samples, we were able to detect both $28 \mathrm{~S}$ and $18 \mathrm{~S}$ ribosomal RNA bands with a density ratio of $\sim 2: 1$. No further analysis was performed on any sample with the $260 / 280$ and the $260 / 230$ ratios below 1.8 or apparently degraded RNA. Samples were stored at $-80{ }^{\circ} \mathrm{C}$.

Reverse transcription was carried out using Superscript III One-Step RT-PCR System (Invitrogen) on equal amounts of total RNA $(0.5 \mu \mathrm{g})$. Negative controls were performed in parallel by omitting RNA or enzyme.

\subsection{Real-time quantitative $R T-P C R-R T-q P C R$}

We analyzed the expression of GAPDH, HPRT1, 18s rRNA, GUSB and ACTB by quantitative PCR using Taqman Gene Expression Assays (Applied Biosystems) following the manufacturer's protocol on iCycler IQ detection system (Bio-Rad). The PCR reaction efficiency for each gene assay was tested using 2-fold serial dilutions (from 50 to $3.125 \mathrm{ng}$ ) of cDNAs randomly chosen among the samples. Each set of primers and probe showed an efficiency of $90-100 \%$. All calibration curves exhibited correlation coefficients higher than 0.99. Assays were performed in duplicate with cDNA equivalent to $10 \mathrm{ng}$ of reverse transcribed RNA.

The absence of genomic DNA contamination was confirmed by PCR amplification of RNA samples in the absence of cDNA synthesis. The threshold cycles (CTs) were determined and converted to expression values $(R)$ by the following equation: $R=E^{-C T}$ where $E$ was the efficiency of each sets of primers/probe.
Taqman Gene Expression Assays IDs for each set of primers and probe were as follows: Hs99999905m1 (GAPDH) and Hs99999905m1 (HPRT1). GUSB, ACTB and 18s rRNA endogenous controls were from Applied Biosystems.

\subsection{Data analysis}

The expression of transcripts, where the highest relative quantity for each gene was set to 1 , was analyzed by investigating standard statistical parameters using Microsoft Excel software. The significance of the difference in the mean level for each transcript between the age groups of samples was determined by means of one-way analysis of variance (ANOVA) followed by the Dunnett's post hoc analysis (where a $p$-value $<0.05$ was considered statistically significant).

In order to determine the most stable housekeeping genes among the set of tested genes, a comparison in the variation of gene expression was performed by means of coefficients of variation (CV), calculated as standard deviation/mean, and two Add-in for Microsoft Excel: geNorm (Vandesompele et al., 2002) and NormFinder (Andersen et al., 2004). GeNorm uses an algorithm to calculate $M$ value, a gene expression stability measure, defined as the mean pairwise variation for a given gene compared to the remaining tested genes. Hence, a lower value of $M$ indicates higher stability of the reference gene. We considered 0.5 as a cut-off for $M$ value; genes with a $M$-value above this value are considered unreliable for normalization. The programme also establishes a rank order of gene stability via stepwise exclusion of the least stable gene, which allows identifying the remaining couple of genes having the lowest $M$-value. To determine how many reference genes should be used for accurate normalization, geNorm also performs a stepwise calculation between sequential normalization factors (NF). It starts to calculate the pairwise variation $V_{2 / 3}$ between the NF2 (including the two most stable reference genes) and the normalization factor NF3 (including the three most stable reference genes). Then, it performs a stepwise calculation of the $V_{n / n+1}$ between the $\mathrm{NF}_{n}$ and the $\mathrm{NF}_{n+1}$. A variation of the $V_{n / n+1}$ above 0.15 indicates that the inclusion of an additional reference gene is required.

NormFinder, whose strategy is rooted in a mathematical model of gene expression, provides a ranking of the tested genes based on a direct measure of both the overall variation of expression of candidate reference genes in the samples group and the variation between samples subgroups. The combined measure of intra- and intergroup-variation is given as a stability value which is an estimation of the variation in expression of candidate reference genes. Low stability values define genes showing high stability of expression. In our settings the intragroup variation is calculated across all samples whether the intergroup is calculated between the 8 age groups of samples.

\section{Results}

\subsection{Levels of candidate reference genes $m R N A$}

The distribution of the expression levels - obtained from CT values using the Pfaffl method (Pfaffl, 2001) - is given in Fig. 1.18s rRNA showed the most abundant level of expression reaching about 200,000-fold difference in comparison to the least expressed genes HPRT1 and GUSB.

With the exception of $18 \mathrm{~s}$, which showed a significant downregulation in the last age group (70-74), all other candidate genes did not show any significant difference in the mean expression across the different age groups.

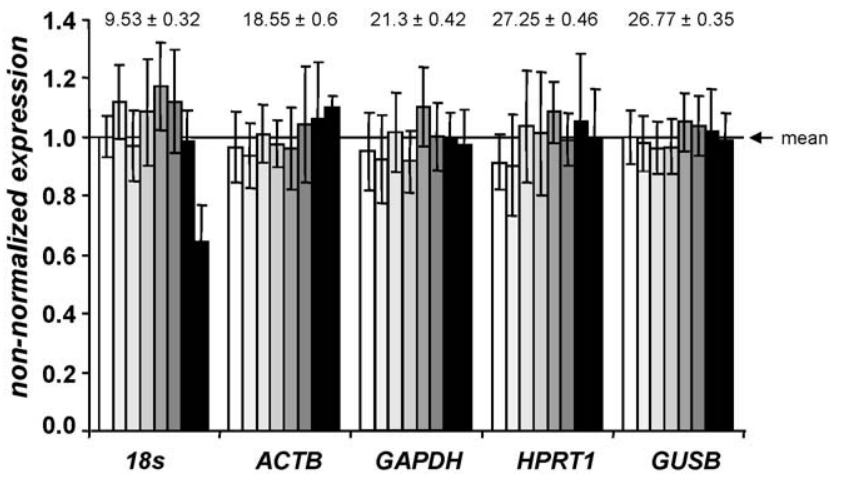

Fig. 1. Levels of candidate reference genes expression. Histogram shows mean levels of candidate genes expression starting from equal amounts of RNA obtained in 8 age-stratified 5 subjects groups. Values are given as mean \pm SD. The mean expression level across all samples was arbitrarily set as 1.0. Numbers indicate the average threshold cycle \pm SD measured across all samples. 


\subsection{Expression stability of putative reference genes}

A commonly accepted feature defining a candidate housekeeping gene is the stability of its expression, i.e. a small CV, a maximum fold change $<2$ (MFC, the ratio of the maximum and minimum values observed within the dataset) and a mean expression level lower than the maximum expression level subtracted of two standard deviation (SD).

The levels of the five-candidate genes expression in a set of 40 PBMCs samples from donors in the age range of 35-74 years were used. Table 1 shows the ranking of the tested housekeeping genes in order of increasing CV. A lower CV value (defined as the ratio of the standard deviation to the average expression) corresponds to a higher intergroup stability. GUSB and GAPDH showed a considerably higher stability with respect to $18 \mathrm{~s}, A C T B$ and HPRT1. In particular GUSB ranked in the first position regarding expression stability. All genes had a MFC below 2, in contrast the mean expression was slightly higher than the maximum expression level minus 2 standard deviations in all cases.

The expression stability of the selected housekeeping genes was further addressed using the geNorm and the NormFinder softwares. GeNorm estimates the stability value $M$ using a pairwise comparison approach ranking putative housekeeping genes according to the similarity of expression profiles across the sample set. The $M$-value was calculated for each gene over all 40 samples (total age range analysis) to find the most stable gene (lower $M$ value). All the investigated genes exhibited high expression stability with $M$-values below the algorithm defined cut-off value of 0.5 (Allen et al., 2008; Silver et al., 2008; Vandesompele et al., 2002). The tested transcripts are ranked according to their stability in Table 2 . The average $M$-values calculated by stepwise exclusion of the least stable gene indicated that GUSB and GAPDH are the most stable reference genes, with combined $M$-value equal to 0.21 (Fig. 2).

The optimal number of reference genes which should be used for accurate normalization was determined by calculating the normalization factor (NF). This is calculated by geNorm from two or more genes with the variable $V$ as pairwise variation $\left(V_{n} / V_{n+1}\right)$ between two sequential normalization factors $\left(\mathrm{NF}_{n}\right.$ and $\left.\mathrm{NF}_{n+1}\right)$. The use of more than the two most stable reference genes identified (GAPDH and GUSB) is not required as suggested by the $V$-value

Table 1

Ranking of candidate reference genes in order of increasing coefficient of variation (CV).

\begin{tabular}{llrll}
\hline Gene name & Mean & \multicolumn{1}{c}{ CV } & MFC & Max-2SD \\
\hline GUSB & 0.68 & 5.99 & 1.16 & 0.65 \\
GAPDH & 0.61 & 8.23 & 1.26 & 0.60 \\
$18 \mathrm{~s}$ & 0.76 & 10.89 & 1.46 & 0.66 \\
ACTB & 0.57 & 11.44 & 1.43 & 0.50 \\
HPRT1 & 0.61 & 13.54 & 1.46 & 0.55 \\
\hline
\end{tabular}

$\mathrm{CV}$ equals the standard deviation (SD) divided by the mean (expressed as percentage). MCF, the maximum fold change; Max-2SD, maximum expression level subtracted of 2SD.

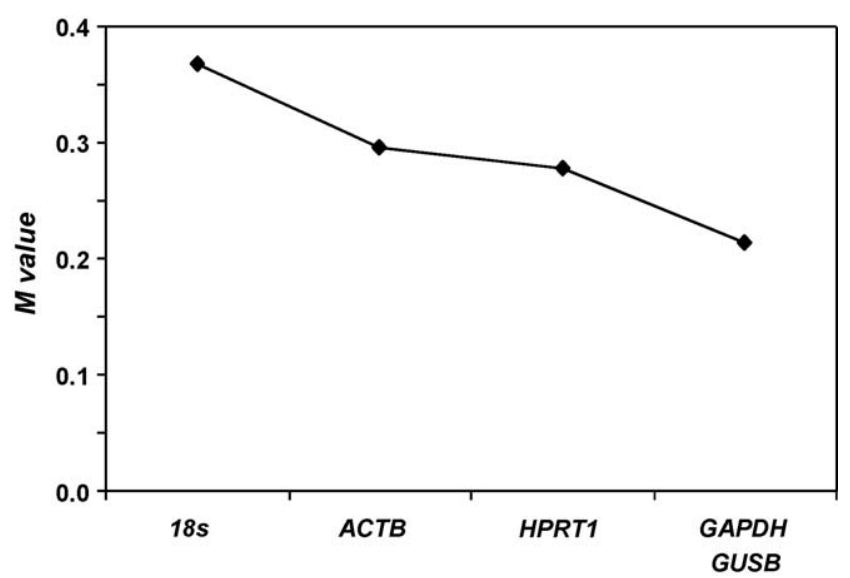

Fig. 2. Selection of the best pair of candidate reference genes for accurate normalization by geNorm. The average expression stability $M$ of candidate reference genes during stepwise exclusion of the least stable gene is shown. The $X$-axis indicates genes ranked according to their expression stability measure. The $Y$-axis indicates the average $M$-value of the remaining genes after having excluded the indicated gene (i.e. the least stable).

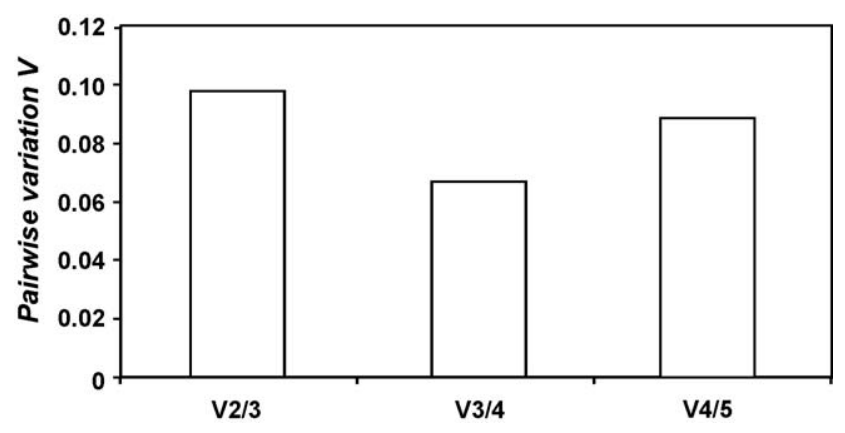

Fig. 3. Optimal number of reference genes for accurate normalization as determined by geNorm. Pairwise variation between two sequential normalization factors $\left(\mathrm{NF}_{n}\right.$ and $\mathrm{NF}_{n+1}$ ) to determine the optimal number of reference genes for reliable normalization.

below the cut-off 0.15 which has been indicated by authors as the limit beneath which it would not be necessary to include additional reference genes (Vandesompele et al., 2002) (Fig. 3).

As co-regulation of housekeeping genes could influence the efficiency of geNorm analysis, the alternative software NormFinder was used in parallel. The results of NormFinder, which ranks housekeeping genes according to the least estimated intra- and intergroup gene expression variation (lower stability value), confirmed exactly geNorm analysis both in the ranking order of housekeeping genes and in the best combination of genes to use for accurate normalization (Table 3 ).

Gender could be an additional factor affecting gene expression stability. In particular it is known that the expression levels of some commonly used housekeeping genes is differentially
Table 2

Expression stability measure $(M)$ calculated by geNorm for each candidate reference gene over all 40 samples.

\begin{tabular}{ll}
\hline Gene name & M-Value \\
\hline GUSB & 0.310 \\
GAPDH & 0.330 \\
HPRT1 & 0.347 \\
ACTB & 0.375 \\
$18 \mathrm{~s}$ & 0.476 \\
\hline
\end{tabular}

Lower $M$-value indicates higher expression stability.
Table 3

Expression stability value calculated by NormFinder for each candidate reference gene over all 40 samples.

\begin{tabular}{ll}
\hline Gene name & Stability value \\
\hline GAPDH + GUSB & 0.031 \\
GUSB & 0.035 \\
GAPDH & 0.043 \\
HPRT1 & 0.053 \\
ACTB & 0.054 \\
$18 S$ & 0.083 \\
\hline
\end{tabular}

A lower value indicates higher expression stability. 
regulated in gender-specific manner (Verma and Shapiro, 2006). Although each age subgroup previously analyzed was composed of the two genders represented homogeneously (2 men and 3 women, with the exception of the 45-49 and 65-69 age groups which were composed of 3 men and 2 women), we decided to assess whether the composition of our population of samples could affect the selection of an age-indifferent control gene. To this aim the analysis of transcript stability already performed for the gender-mixed group of samples was compared to the analysis with the samples regrouped for gender. As shown in supplementary Tables 1 and 2, geNorm and NormFinder analyses gave the same ranking order either when male and female groups are compared or when both groups are compared with the gender-mixed group of samples (Tables 2 and 3). However we noted that the absolute values for the stability parameters were higher for females than for males. This finding indicated that the contribution of males and females to the stability of tested genes expression was quantitatively different. Nevertheless, no significant gene-specific alteration of the expression stability was detectable. So the mixed composition of our population of samples did not affect the comparison of the tested genes in terms of stability of gene expression.

With the aim to assess further gene expression stability with respect to age both geNorm and NormFinder analyses were carried out on the samples regrouped into six overlapping age subgroups each containing 15 samples. As shown in Fig. 4, gene expression stability of candidate reference genes, with the exception of GUSB, was not homogeneous across the samples of the different age subgroups. In particular geNorm $M$-value for $18 \mathrm{~s}, A C T B$ and HPRT1 revealed a gradual loss of expression stability with increasing age. Gene expression stability for $18 \mathrm{~s}$ was particularly affected in the last group of samples with age spanning from 60 to 74 years where the $M$-value overcame the limit of 0.5 for accurate normalization (Fig. 4A). This age-related gain of gene expression variation was also detected by NormFinder for $A C T B$ and $18 \mathrm{~s}$ (Fig. 4B). In contrast
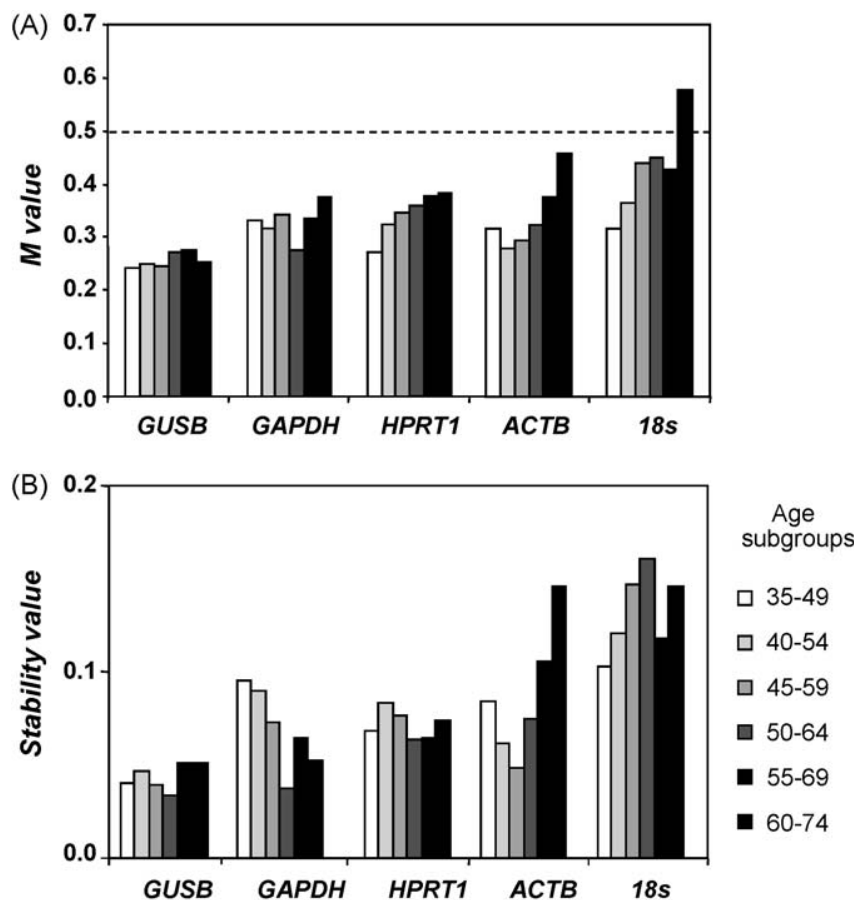

Fig. 4. Expression stability of candidate reference genes across groups of samples of different age. GeNorm stability measure $M(\mathrm{~A})$ and NormFinder stability value (B) calculated over the samples regrouped into 6 overlapping age subgroups each composed by 15 samples. Dashed line in (A) indicates the cut-off for $M$-value.
NormFinder detected an opposite behavior of GAPDH whose stability value decreased with increasing age revealing a gain of gene expression stability with the aging process. Both approaches indicated GUSB as the housekeeping gene having the most homogeneous expression stability across the different age groups.

\subsection{Validation of putative reference genes}

To validate the stability-based ranking of the putative control genes under investigation, we monitored the significance of the difference in the mean expression of a target gene between the age subgroups upon normalization with different control genes. To this purpose, the levels of $18 \mathrm{~s}$ gene expression were determined using GUSB, GAPDH, HPRT1 and ACTB as control genes. As was shown in Fig. 1, a significant down-regulation of $18 \mathrm{~s}$ transcript expression was detectable in the last age group (70-74) when raw data of expression across age subgroups were compared. By the use of the one-way analysis of variance (ANOVA) we compared the degree of dispersion of $18 \mathrm{~s}$ normalized expression within the age groups with respect to the total amount of dispersion in the samples. As shown in Fig. 5, the capability of the control genes in making the difference in the mean expression across age groups of $18 \mathrm{~s}$ transcript significant was strikingly different. This was evidenced by the observation that the $F$-values increased in accordance to the stability measured previously for each control gene (see Tables 2 and 3). When normalizing to the least stable housekeeping genes ( $A C T B$, HPTR1 and GAPDH) no significant difference between age groups was revealed $(F<$ Fcrit; Fcrit $=2.42$ at $p<0.05$ ). In contrast, when using the most stable GUSB as a reference gene, a statistically significant difference between the age groups could be observed ( $F>$ Fcrit). Furthermore, by comparing GUSB-normalized $18 \mathrm{~s}$ expression measured in each age group against the first group (35-39) by the Dunnett's post hoc analysis, we found a significant decrement of $18 \mathrm{~s}$ expression in the last age group (70-74) $(p<0.05)$

To provide further evidence about the stability of selected reference genes in an independent dataset, we next tested the freely accessible Gene Aging Nexus (GAN) database containing results from microarray experiments addressed to reveal agingrelated gene expression patterns (Pan et al., 2007). From the GAN database we collected data of an extensive microarray gene expression profiling of human brain at various ages. This array analysis was performed on the postmortem frontal cortex of 18 normal males and 12 normal females at 26-106 years of age (see supplementary data for more information). As shown in supplementary Table 3, none of the transcripts tested - i.e. GUSB, GAPDH, HPRT1 and ACTB (N.B. 18s rRNA was not available in the arrays) was found to be modulated by the aging process. Furthermore the analysis of expression stability - by the calculation of the CV - of GUSB, GAPDH, HPRT1 and ACTB confirmed the results obtained from our dataset using geNorm and NormFinder. In particular the ranking of the tested genes in order of decreasing CV was exactly superimposable to the stability ranking that was obtained from our set of PBMCs samples. GUSB appeared to be the least variable transcript followed by GAPDH, HPRT1 and ACTB. In addition the CV of the putative reference genes appeared to be significantly lower than the $\mathrm{CV}$ of some differentially expressed genes (for example ABCG1 or APBA1, supplementary Table 3 ).

To gain evidence of the suitability of the tested genes for accurate normalization we normalized the expression of $A B C G 1$ transcript, whose level was found to be up-regulated in the elderly individuals, to GUSB and $A C T B$, respectively, the least and the most variable housekeeping gene being tested. As shown in supplementary figure, when the reference gene was GUSB a significant difference in the $A B C G 1$ transcript mean level was revealed between sample groups of increasing age $(F>$ Fcrit; Fcrit $=3.34$ at 

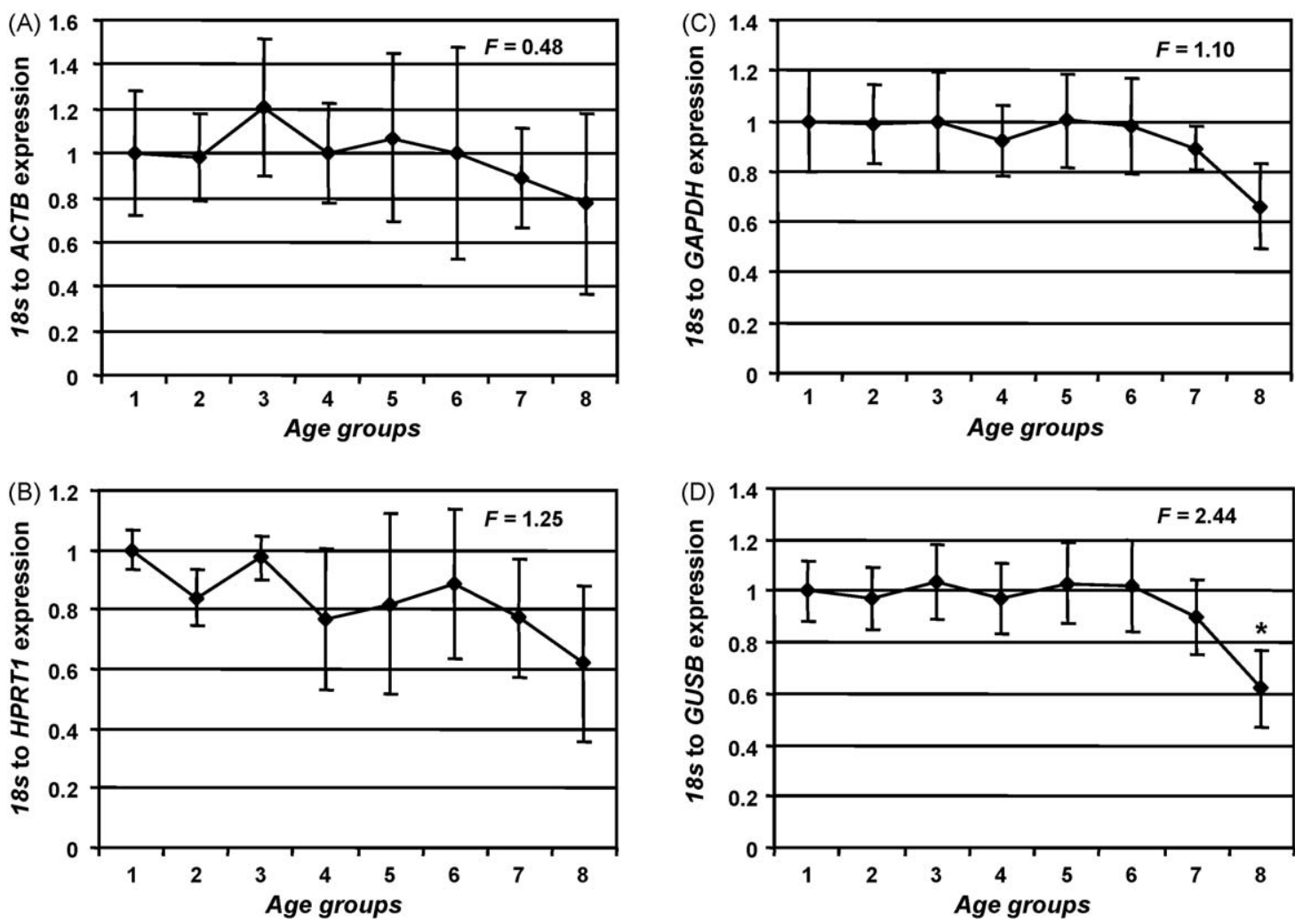

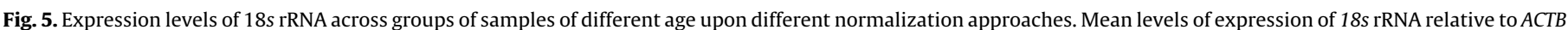

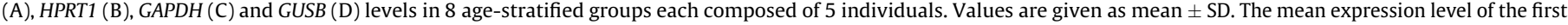
group of samples was arbitrarily set as 1.0 . ( $F$, ANOVA $F$ value; Fcrit $=2.42$ at $p<0.05 ;{ }^{*} p<0.05$ against the first group of age after Dunnett's test).

$p<0.05)$. In contrast when using the least stable $A C T B$ transcript, the induction of $A B C G 1$ in the aged groups of samples was not supported by statistical evidence due to an increased variation within samples groups $(F<F c r i t)$.

\section{Discussion}

The analysis of gene expression profile has become pivotal to biomedical research as transcriptional regulation is an important mechanism mediating cellular responses to various stimuli. To date, real-time quantitative RT-PCR is the gold standard method by which a specific and highly sensitive determination of target gene expression could be achieved. Despite being a powerful, mainstream research tool, it is not a standardized technology. In particular, the reliability of quantitative PCR measurements is highly dependent on a careful experimental design aimed to obtain acceptable reproducibility and biological validity of mRNA quantifications (Bustin, 2000, 2002; Bustin and Nolan, 2004). One of the most important problems affecting the accuracy of quantitative expression data is the choice of an appropriate normalization strategy. Worryingly, this is still a not widely appreciated or acknowledged step in gene quantification analysis and therefore requires targeted experimental investigation. There is no universally accepted strategy for normalization as there is no error-free procedure (Huggett et al., 2005). The coamplification of a single "reference gene" as invariant endogenous control in the assay is the most widely adopted procedure of normalization. It corrects target gene expression data for non-biological sample-tosample variations due to non-homogeneous qualities of starting RNA, RT-PCR efficiency, and errors in sample quantification (Huggett et al., 2005; Pfaffl, 2001; Pfaffl and Hageleit, 2001; Dheda et al., 2004). However, this procedure can be dramatically misleading when the reference gene is selected without taking into account its specific sample-to-sample biological variation of expression. The common practice of electing an housekeeping gene as reference based on the presumption of its invariable expression has been clearly discredited by a number of papers (de Jonge et al., 2007; Goidin et al., 2001; Ross et al., 2000; Vandesompele et al., 2002). Increasing evidences show that the mRNA level of commonly used housekeeping genes is not constant among individuals, tissues or experimental conditions. Nevertheless, the majority of reported research involving gene expression analysis adopts the risky, biased procedure of using a nonvalidated reference gene. Since it is to assume that a universal, absolute standard for normalization is probably unavailable, for the purpose of an unbiased comparison of mRNA transcription profiles, it is crucial to select the most suitable transcript to meet the criteria of invariant reference gene as closely as possible.

Aging is characterized by drastic, tissue-specific functional alterations and changes of gene expression profiles. Variation in gene expression among individuals tends to increase with age and it has been clearly shown both in human and mouse that the aging process is a confounding factor with respect to expression stability of some housekeeping genes (Bahar et al., 2006; Boda et al., 2009; Sieber et al., 2008; Somel et al., 2006; Tanic et al., 2007; Touchberry et al., 2006).

To our knowledge, this is the first study that evaluates the suitability of potential reference genes for gene expression analysis in human aging. In this study, five pre-selected bona fide invariantly transcribed housekeeping genes, used in numerous previous studies as reference genes (Suzuki et al., 2000), were tested in PBMCs from 40 individuals in order to investigate the impact of aging on their expression stability. There is no unambiguous procedure to estimate gene expression variation as there is no agreement of opinions on how to define invariant gene expression when comparing raw data of expression 
(Andersen et al., 2004). To gain an unbiased estimation of the stability of the selected candidate reference genes we adopted a multiple analytical approach. The pairwise comparison approach (geNorm) selects the most suitable reference gene on the basis of the variation of expression ratios between candidate reference genes expression across the sample set. It is based on the assumption that the ratio between two putative reference genes is constant across samples independently to RNA amount analyzed per sample. Therefore, the variation of this ratio for two candidate reference genes across samples (pairwise variation) is a measure of gene stability. However, geNorm algorithm analysis, being very robust against sampling errors, is based on the assumption that none of the genes analyzed in this study are co-regulated. Although the genes analyzed in this study are involved in diverse cellular functions, co-regulation between them cannot be ruled-out since the aging process has a broad effect on expression of multiple functional classes of genes. Hence, the stability of candidate reference genes was also analyzed by simply evaluating the dispersion of measured mRNA levels by calculating the CV and the "stability value" by NormFinder algorithm. These last approaches of general variation analysis could be sensitive to sampling errors and outliners but are less affected by the eventuality of coregulation. All the strategies we used in parallel yielded data indicating GUSB, followed in the ranking by GAPDH, to be the gene showing the most invariant and age-indifferent gene expression among the candidates evaluated. Interestingly, this finding confirmed in humans PBMCs what has been shown in the mouse white blood cells where GUSB and GAPDH have been selected among some other common housekeeping genes as relatively stable control genes with respect to aging (Sieber et al., 2008). Similarly GUSB and GAPDH seemed to be relatively stable against aging also in other tissues as it has been shown in the mouse and rat brain tissues (Boda et al., 2009; Tanic et al., 2007).

Although GAPDH, HPRT1 and ACTB - with the exception of $18 \mathrm{~s}$ rRNA - showed an absolute gene expression stability which was predicted to be sufficient for accurate normalization, their stability was clearly influenced by the aging process. This last evidence suggests a further homogeneity criterion that has to be considered when choosing a reference gene for accurate normalization. The gradual loss of expression stability with increasing age here reported for $A C T B, H P R T 1$ and $18 \mathrm{~s}$ is reminiscent of the agecorrelated heterogeneity of expression (ACHE) (Bahar et al., 2006; Somel et al., 2006).

Another intriguing finding we are describing here is the downregulation of $18 \mathrm{~s}$ rRNA occurring in the last age group of individuals (70-74). Our data are in agreement with the findings of Boda et al. (2009) showing a significantly lower level of $18 \mathrm{~s}$ expression in the adult mouse hemibrain in comparison to the young one. Moreover an age-related dysfunction of rRNA synthesis has been addressed by a number of studies, many of which indicated that there is a selective loss of the rDNA (Gaubatz and Cutler, 1978; Johnson and Strehler, 1972; Strehler and Chang, 1979; Strehler et al., 1979) or hypermethylation (Swisshelm et al., 1990) during the course of aging.

In conclusion, results from this study indicate that putative housekeeping genes cannot be presumed to be stably expressed in the same tissue type with respect to the aging process and that their validation as reference genes is mandatory. The procedure for the selection of a reference gene here presented could serve as guideline for researchers aiming to perform accurate RT-PCR expression profiling, which opens up the possibility of studying the biological relevance of expression differences among differently aged individuals. Thus we provide evidence for GUSB to be the most suitable reference gene among the analyzed genes for expression studies based on RT-quantitative PCR in human PBMCs with age as variable.

\section{Acknowledgements}

This work was supported by the European Union FP7 Health Research, grant number HEALTH-F4-2008-200880 (Large-scale integrating project MARK-AGE). We thank Gudrun von Scheven for technical assistance.

\section{Appendix A. Supplementary data}

Supplementary data associated with this article can be found, in the online version, at doi:10.1016/j.mad.2009.12.005.

\section{References}

Allen, D., Winters, E., Kenna, P.F., Humphries, P., Farrar, G.J., 2008. Reference gene selection for real-time rtPCR in human epidermal keratinocytes. J. Dermatol. Sci. 49, 217-225.

Andersen, C.L., Jensen, J.L., Orntoft, T.F., 2004. Normalization of real-time quantitative reverse transcription-PCR data: a model-based variance estimation approach to identify genes suited for normalization, applied to bladder and colon cancer data sets. Cancer Res. 64, 5245-5250.

Bahar, R., Hartmann, C.H., Rodriguez, K.A., Denny, A.D., Busuttil, R.A., Dolle, M.E., Calder, R.B., Chisholm, G.B., Pollock, B.H., Klein, C.A., Vijg, J., 2006. Increased cellto-cell variation in gene expression in ageing mouse heart. Nature 441, 10111014.

Bas, A., Forsberg, G., Hammarstrom, S., Hammarstrom, M.L., 2004. Utility of the housekeeping genes 18S rRNA, beta-actin and glyceraldehyde-3-phosphatedehydrogenase for normalization in real-time quantitative reverse transcriptase-polymerase chain reaction analysis of gene expression in human $\mathrm{T}$ lymphocytes. Scand. J. Immunol. 59, 566-573.

Boda, E., Pini, A., Hoxha, E., Parolisi, R., Tempia, F., 2009. Selection of reference genes for quantitative real-time RT-PCR studies in mouse brain. J. Mol. Neurosci. 37 $238-253$.

Burdge, G.C., Slater-Jefferies, J., Torrens, C., Phillips, E.S., Hanson, M.A., Lillycrop, K.A. 2007. Dietary protein restriction of pregnant rats in the F0 generation induces altered methylation of hepatic gene promoters in the adult male offspring in the F1 and F2 generations. Br. J. Nutr. 97, 435-439.

Bustin, S.A., 2000. Absolute quantification of mRNA using real-time reverse transcription polymerase chain reaction assays. J. Mol. Endocrinol. 25, 169-193.

Bustin, S.A., 2002. Quantification of mRNA using real-time reverse transcription PCR, RT-PCR: trends and problems. J. Mol. Endocrinol. 29, 23-39.

Bustin, S.A., Nolan, T., 2004. Pitfalls of quantitative real-time reverse-transcription polymerase chain reaction. J. Biomol. Tech. 15, 155-166.

Calvanese, V., Lara, E., Kahn, A., Fraga, M., 2009. The role of epigenetics in aging and age related diseases. Ageing Res. Rev, 8, 268-276.

Campisi, J., Kim, S.H., Lim, C.S., Rubio, M., 2001. Cellular senescence, cancer and aging: the telomere connection. Exp. Gerontol. 36, 1619-1637.

de Jonge, H.J., Fehrmann, R.S., de Bont, E.S., Hofstra, R.M., Gerbens, F., Kamps, W.A de Vries, E.G., van der Zee, A.G., te Meerman, G.J., ter Elst, A., 2007. Evidence based selection of housekeeping genes. PLoS One 2, e898.

Delage, B., Dashwood, R.H., 2008. Dietary manipulation of histone structure and function. Annu. Rev. Nutr. 28, 347-366.

Dheda, K., Huggett, J.F., Bustin, S.A., Johnson, M.A., Rook, G., Zumla, A., 2004. Validation of housekeeping genes for normalizing RNA expression in real-time PCR. Biotechniques 37 (116), 118-119 112-4

Feil, R., 2006. Environmental and nutritional effects on the epigenetic regulation of genes. Mutat. Res. 600, 46-57.

Feinberg, A.P., 2007. Phenotypic plasticity and the epigenetics of human disease. Nature 447, 433-440.

Finkel, T., Holbrook, N.J., 2000. Oxidants, oxidative stress and the biology of ageing. Nature 408, 239-247.

Fraga, M.F., 2009. Genetic and epigenetic regulation of aging. Curr. Opin. Immunol. 21, 446-453.

Fraga, M.F., Agrelo, R., Esteller, M., 2007. Cross-talk between aging and cancer: the epigenetic language. Ann. N.Y. Acad. Sci. 1100, 60-74.

Fraga, M.F., Ballestar, E., Paz, M.F., Ropero, S., Setien, F., Ballestar, M.L., Heine-Suner, D., Cigudosa, J.C., Urioste, M., Benitez, J., Boix-Chornet, M., Sanchez-Aguilera, A Ling, C., Carlsson, E., Poulsen, P., Vaag, A., Stephan, Z., Spector, T.D., Wu, Y.Z., Plass, C., Esteller, M., 2005. Epigenetic differences arise during the lifetime of monozygotic twins. Proc. Natl. Acad. Sci. U.S.A. 102, 10604-10609.

Gaubatz, J., Cutler, R.G., 1978. Age-related differences in the number of ribosomal RNA genes of mouse tissues. Gerontology 24, 179-207.

Ghoshal, K., Li, X., Datta, J., Bai, S., Pogribny, I., Pogribny, M., Huang, Y., Young, D. Jacob, S.T., 2006. A folate- and methyl-deficient diet alters the expression of DNA methyltransferases and methyl CpG binding proteins involved in epigenetic gene silencing in livers of F344 rats. J. Nutr. 136, 1522-1527.

Johnson, R., Strehler, R.L., 1972. Loss of genes coding for ribosomal RNA in ageing brain cells. Nature 240, 412-414.

Goidin, D., Mamessier, A., Staquet, M.J., Schmitt, D., Berthier-Vergnes, O., 2001 Ribosomal 18s RNA prevails over glyceraldehyde-3-phosphate dehydrogenase and beta-actin genes as internal standard for quantitative comparison of mRNA levels in invasive and noninvasive human melanoma cell subpopulations. Anal. Biochem. 295, 17-21. 
Gronbaek, K., Hother, C., Jones, P.A., 2007. Epigenetic changes in cancer. Apmis 115, 1039-1059.

Grube, K., Burkle, A., 1992. Poly(ADP-ribose) polymerase activity in mononuclear leukocytes of 13 mammalian species correlates with species-specific life span. Proc. Natl. Acad. Sci. U.S.A. 89, 11759-11763.

Grummt, I., Ladurner, A.G., 2008. A metabolic throttle regulates the epigenetic state of rDNA. Cell 133, 577-580.

Herceg, Z., 2007. Epigenetics and cancer: towards an evaluation of the impact of environmental and dietary factors. Mutagenesis 22, 91-103.

Huggett, J., Dheda, K., Bustin, S., Zumla, A., 2005. Real-time RT-PCR normalisation; strategies and considerations. Genes Immun. 6, 279-284.

Jaenisch, R., Bird, A., 2003. Epigenetic regulation of gene expression: how the genome integrates intrinsic and environmental signals. Nat. Genet. 33 (Suppl.), 245-254.

Liu, H., Zhou, Y., Boggs, S.E., Belinsky, S.A., Liu, J., 2007. Cigarette smoke induces demethylation of prometastatic oncogene synuclein-gamma in lung cancer cells by downregulation of DNMT3B. Oncogene 26, 5900-5910.

Migliore, L., Coppede, F., 2008. Genetics, environmental factors and the emerging role of epigenetics in neurodegenerative diseases. Mutat. Res. 667, 82-97.

Pan, F., Chiu, C.H., Pulapura, S., Mehan, M.R., Nunez-Iglesias, J., Zhang, K., Kamath, K., Waterman, M.S., Finch, C.E., Zhou, X.J., 2007. Gene Aging Nexus: a web database and data mining platform for microarray data on aging. Nucleic Acids Res. 35, D756-D759.

Petronis, A., Gottesman, I.I., Kan, P., Kennedy, J.L., Basile, V.S., Paterson, A.D. Popendikyte, V., 2003. Monozygotic twins exhibit numerous epigenetic differences: clues to twin discordance? Schizophr. Bull. 29, 169-178.

Pfaffl, M.W., 2001. A new mathematical model for relative quantification in realtime RT-PCR. Nucleic Acids Res. 29, e45.

Pfaffl, M.W., Hageleit, M., 2001. Validities of mRNA quantification using recombinant RNA and recombinant DNA external calibration curves in real-time RTPCR. Biotechnol. Lett. 23, 275-282.

Ross, D.T., Scherf, U., Eisen, M.B., Perou, C.M., Rees, C., Spellman, P., Iyer, V., Jeffrey, S.S., Van de Rijn, M., Waltham, M., Pergamenschikov, A., Lee, J.C., Lashkari, D., Shalon, D., Myers, T.G., Weinstein, J.N., Botstein, D., Brown, P.O., 2000. Systematic variation in gene expression patterns in human cancer cell lines. Nat. Genet. 24, 227-235.

Sieber, M.W., Guenther, M., Kohl, M., Witte, O.W., Claus, R.A., Frahm, C., 2008. Interage variability of bona fide unvaried transcripts. Normalization of quantitative PCR data in ischemic stroke. Neurobiol. Aging [Epub ahead of print].
Silver, N., Cotroneo, E., Proctor, G., Osailan, S., Paterson, K.L., Carpenter, G.H., 2008. Selection of housekeeping genes for gene expression studies in the adult rat submandibular gland under normal, inflamed, atrophic and regenerative states. BMC Mol. Biol. 9, 64.

Somel, M., Khaitovich, P., Bahn, S., Paabo, S., Lachmann, M., 2006. Gene expression becomes heterogeneous with age. Curr. Biol. 16, R359-R360.

Strehler, B.L., Chang, M.P., 1979. Loss of hybridizable ribosomal DNA from human post-mitotic tissues during aging. II. Age-dependent loss in human cerebral cortex-hippocampal and somatosensory cortex comparison. Mech. Ageing Dev. 11, 379-382.

Strehler, B.L., Chang, M.P., Johnson, L.K., 1979. Loss of hybridizable ribosomal DNA from human post-mitotic tissues during aging. I. Age-dependent loss in human myocardium. Mech. Ageing Dev. 11, 371-378.

Sutherland, J.E., Costa, M., 2003. Epigenetics and the environment. Ann. N.Y. Acad. Sci. 983, 151-160.

Suzuki, T., Higgins, P.J., Crawford, D.R., 2000. Control selection for RNA quantitation. Biotechniques 29, 332-337.

Swisshelm, K., Disteche, C.M., Thorvaldsen, J., Nelson, A., Salk, D., 1990. Age-related increase in methylation of ribosomal genes and inactivation of chromosomespecific rRNA gene clusters in mouse. Mutat. Res. 237, 131-146.

Tanic, N., Perovic, M., Mladenovic, A., Ruzdijic, S., Kanazir, S., 2007. Effects of aging, dietary restriction and glucocorticoid treatment on housekeeping gene expression in rat cortex and hippocampus-evaluation by real time RT-PCR. J. Mol. Neurosci. 32, 38-46.

Touchberry, C.D., Wacker, M.J., Richmond, S.R., Whitman, S.A., Godard, M.P., 2006. Age-related changes in relative expression of real-time PCR housekeeping genes in human skeletal muscle. J. Biomol. Tech. 17, 157-162.

Vandesompele, J., De Preter, K., Pattyn, F., Poppe, B., Van Roy, N., De Paepe, A., Speleman, F., 2002. Accurate normalization of real-time quantitative RT-PCR data by geometric averaging of multiple internal control genes. Genome. Biol. 3 RESEARCH0034

Valente, V., Teixeira, S.A., Neder, L., Okamoto, O.K., Oba-Shinjo, S.M., Marie, S.K.N., Scrideli, C.A., Paçó-Larson, M.L., Carlotti, C., 2009. Selection of suitable housekeeping genes for expression analysis in glioblastoma using quantitative RTPCR. BMC Mol. Biol. 10, 17.

Verma, A.S., Shapiro, B.H., 2006. Sex-dependent expression of seven housekeeping genes in rat liver. J. Gastroenterol. Hepatol. 21, 1004-1008.

Waterland, R.A., 2009. Is epigenetics an important link between early life events and adult disease? Horm. Res. 71 (Suppl. 1), 13-16. 